\title{
Identification of Potential Therapeutic Targets in the Liver of Pioglitazone-Treated Type 2 Diabetes Sprague-Dawley Rats via Expression Profile Chip and iTRAQ Assay
}

\author{
Zhong-Xia Lu $\mathbb{D},{ }^{1}$ Wen-Jun Xu $\mathbb{D}^{1},{ }^{1}$ Yang-Sheng Wu $\mathbb{D},{ }^{2}$ Chang-Yu Li $\mathbb{D}^{2},{ }^{2}$ and Yi-Tao Chen $\mathbb{D}^{1}$ \\ ${ }^{1}$ College of Life Sciences, Zhejiang Chinese Medical University, Hangzhou 310053, China \\ ${ }^{2}$ College of Pharmacy, Zhejiang Chinese Medical University, Hangzhou 310053, China
}

Correspondence should be addressed to Chang-Yu Li; lcyzcmu@sina.com and Yi-Tao Chen; cytworld@zcmu.edu.cn

Received 5 October 2017; Accepted 14 January 2018; Published 18 March 2018

Academic Editor: Secundino Cigarran

Copyright ( 2018 Zhong-Xia Lu et al. This is an open access article distributed under the Creative Commons Attribution License, which permits unrestricted use, distribution, and reproduction in any medium, provided the original work is properly cited.

\begin{abstract}
The aim of the present study was to identify key antidiabetic nodes in the livers of pioglitazone-treated type 2 diabetes mellitus Sprague-Dawley rats by transcriptomic and proteomic analysis. Rats were randomly divided into the control, the diabetes model, and the pioglitazone-treated groups. After treatment with pioglitazone for 11 weeks, the effects on fasting blood glucose, body weight, and blood biochemistry parameters were evaluated. Microarray and ITRAQ analysis were used to determine the differentially expressed genes/proteins in rat livers. 1.5-fold changes in gene expression and 1.2-fold changes in protein were set as the screening criteria. After treatment with pioglitazone for 11 weeks, fasting blood glucose in pioglitazone-treated rats was significantly lower than that in the model group. There was a tendency for pioglitazone to reduce TC, TG, TP, ALB, BUN, and HDL-c levels. Kyoto Encyclopedia of Genes and Genomes (KEGG) and gene ontology (GO) were applied to analyze differentially expressed genes/proteins. Furthermore, Western blotting and RT-qPCR were used to validate the results of microarray and iTRAQ. In conclusion, $C y p 7 a 1, C p$, and RT1-EC2 are differentially expressed genes/proteins since they showed a similar trend in rats in the model group and the pioglitazone-treated group.
\end{abstract}

\section{Introduction}

Diabetes mellitus $(\mathrm{DM})$ is a chronic metabolic disease affecting more than 400 million people worldwide [1]. Type 2 diabetes mellitus (T2DM) is caused by insulin resistance and/or insulin deficiency and constitutes almost 95 percent of all diabetes cases [2]. Previous studies have shown that several drugs, including metformin, rosiglitazone, and pioglitazone, can control high blood glucose levels and protect patients from diabetes-related complications [3].

Pioglitazone belongs to the thiazolidinedione class of drugs and was deemed as an insulin sensitizer for the therapy of T2DM [4]. As a highly selective agonist for peroxisome proliferator-activated receptor $\gamma$ (PPAR- $\gamma)$, pioglitazone regulates the transcription of several genes and proteins involved in glucose and lipid metabolism [5]. Shannon et al. reported that pioglitazone inhibited pyruvate-driven
ATP synthesis and hepatocellular glucose production as well as suppressing mitochondrial pyruvate transport regulators, MPC1 and MPC2 [6]. Moreover, the expression of adiponectin is upregulated by pioglitazone [7]. Furthermore, the phosphorylation of AKT is decreased in T2DM mice while receiving pioglitazone treatment [8]. Therefore, it is obvious that pioglitazone is a multitarget and multipathway compound involved in antidiabetic efficacy. However, the key nodes involved in the multitarget antidiabetic effect of pioglitazone still need to be elucidated.

Network pharmacology, which is based on the concept that many drugs act on multiple rather than a single therapeutic target, is recognized as a kind of system biology and network analysis methodology and technology $[9,10]$. Key molecular networks involved in biochemical networks, bioinformatics, and systems biology have been reconstructed, which are helpful in predicting novel targets with high 
efficiency [11-13]. For many drugs, including metformin, morin, and curcumin, several novel candidate targets were identified through network pharmacology assays [14-16].

In the present study, we established type 2 diabetes mellitus rats by injection of streptozotocin (STZ), and the potential therapeutic target nodes in the liver of pioglitazone-treated rats were screened by expression profile chip and iTRAQ assay. Furthermore, metabolic pathways regulated by pioglitazone were also explored.

\section{Materials and Methods}

2.1. Drugs and Reagents. Pioglitazone hydrochloride tablets were purchased from Takeda Pharmaceutical Industry (Osaka, Japan), rat GE 4x44K v3 microarrays and a Gene Expression Hybridization Kit were from Agilent Technologies (Shanghai, China), and QIAGEN RNeasy ${ }^{\circledR}$ Mini Kit was from QIAGEN (Shanghai, China). A prime Script RT Reagent Kit was from TaKaRa Biotechnology (Dalian, China).

2.2. Groups and Treatments. Male SD rats (three per cage, $200 \mathrm{~g}$ average body weight), 8 weeks of age, were obtained from the Animal Center of Zhejiang Chinese Medical University (Hangzhou, China). All animal experiments were carried out in Zhejiang Chinese Medical University Laboratory Animal Research Center (rodent license number SCXK 2013-0115). The experimental protocol was approved by the Ethics Committee of Zhejiang Chinese Medical University.

Before the experimental test, all animals were fed with the basic diet (Zhejiang Academy of Medical Sciences, Hangzhou, China) for 3 weeks. Subsequently, a type 2 diabetes model was established by injection of freshly prepared STZ $(30 \mathrm{mg} / \mathrm{kg})$ dissolved in ice-cold citrate buffer $(0.1 \mathrm{M}$, $\mathrm{pH}$ 4.2). To obtain diabetic symptoms, T2DM rats were fed with high-glucose/high-fat diet, consisting of $69.75 \%$ basic diet, $10 \%$ lard, $10 \%$ yolk powder, $0.25 \%$ cholesterol, and $10 \%$ sucrose (Zhejiang Academy of Medical Sciences, Hangzhou, China), whereas 10 normal rats in the control group were fed with basic diet. Rats with blood glucose at $15-25 \mathrm{mmol} / \mathrm{l}$ were used in the experimental test, which were randomly divided into two groups, the diabetes model group (DM, $n=12$ ) and the pioglitazone-treated group $(\mathrm{n}=12)$. Rats in the control and the model group were given $0.25 \%$ CMC-Na $(10 \mathrm{ml} / \mathrm{kg}$, China National Pharmaceutical Group, Shanghai, China), whereas rats in the treated groups received pioglitazone $(5 \mathrm{mg} / \mathrm{kg}$, in $0.25 \% \mathrm{CMC}-\mathrm{Na})$ via gavage once a day for 11 weeks. All animals were housed at $25^{\circ} \mathrm{C}$ with $50 \sim 70 \%$ humidity and a $12 \mathrm{~h}$ light $/ 12 \mathrm{~h}$ dark cycle with free access to food and water.

2.3. Sample Collection and Index Detection. At the starting ( 0 week) and in the end (11th week) of the experiment, blood was collected from the orbital veins of the rats. The blood was used for the determination of biochemistry parameters, using commercially available kits in a semiauto analyzer (Photometer 5010 V5+, Berlin, Germany). Moreover, after an eight-hour fasting period, blood glucose levels were measured every two weeks using a glucose meter
(Accu-Chek Performa, Roche Diagnostics GmbH, Mannheim, Germany). Body weights were recorded every two weeks. At the end of the experiment, rats were euthanized and livers were quickly dissected on ice. The livers were washed with DEPC water, frozen in liquid nitrogen, and stored at $-80^{\circ} \mathrm{C}$.

2.4. Microarray and iTRAQ Assay. A total of 18 samples ( $n=6$ samples per group) were used for microarray and iTRAQ assay. Total RNA was extracted from liver tissues using the MiniRNeasy Kit based on the manufacturer's instructions (QIAGEN, Shanghai, China). After the determination of the RNA integrity (Agilent Bioanalyzer 2100), RNA was hybridized to a Whole Rat Genome Oligo 4x44 K microarray (Agilent Technologies, Shanghai, China). The significantly differentially expressed genes were screened with a $>1.5$-fold differential expression, and $P<0.05$ was considered statistically significant.

Total liver protein $(100 \mu \mathrm{g})$ from each sample was digested with trypsin, dried, and labeled with different tags per group. Control group samples labeled 114 tags, model group samples labeled 116, and pioglitazone-treated samples labeled 121. The labeled peptides were purified with a strong cation exchange chromatography (SCX) column (Phenomenex, California, USA) and separated by liquid chromatography (LC) using the LC-20AB HPLC Pump system (Shimadzu, Kyoto, Japan). Proteomic data collection was performed using a Triple TOF 5600 system (AB SCIEX, ON, USA) and a pulled quartz tip as the emitter (New Objectives, MA, USA). Proteins that were significantly different were screened with a $>1.2$-fold differential expression, and $P<0.05$ cut-off in statistical analyses.

2.5. GO Enrichment and KEGG Analysis. The GO platform is a bioinformatics resource describing gene product attributes which have three categories, namely, biological process (BP), molecular function (MF), and cellular component (CC) [17]. We defined the significance of GO enrichment according to a $P$ value, with a cut-off value of 0.05 . The KEGG was applied to investigate if target genes were involved in multiple pathways. The top 10 pathway candidates that were potentially related to treatment were selected.

2.6. Reverse Transcription Quantitative Polymerase Chain Reaction (RT-qPCR) Assay. RT-qPCR was used to verify experimental data. Total RNA was extracted as described above, and double-strand cDNA was synthesized according to the manufacturer's instructions (TaKaRa Biotechnology, Shanghai, China). Primers were purchased from Sangon Biological Technology (Shanghai, China), of which the Cyp7a1 former primer was $5^{\prime}$-GCATCTCAAGCAAACACCAT-3', the downstream primer was $5^{\prime}$-TCCACTCACTTCTTCA GAGGC-3', and the amplified fragment was $98 \mathrm{bp}$. Besides, the former primer of $C p$ was $5^{\prime}$-TGATGGCTATGGGC AATGA-3', the downstream primer was $5^{\prime}$ - GGTTTGG TATGTTCCAGGGA-3', and the amplified fragment was $125 \mathrm{bp}$. Data were normalized to the expression level of a $\beta$-actin reference gene, in which upstream primer was $5^{\prime}$-GCTCTCTTCCAGCCTTCCTT-3' and the downstream 
primer was $5^{\prime}$-GGTCTTTACGGATGTCAACG-3'. And its amplified fragment was $105 \mathrm{bp}$. RT-qPCR was performed with a Real Time PCR Machine (Eppendorf Realplex-4, Eppendorf, Germany).

2.7. Western Blotting Assay. Proteins $(20 \mu \mathrm{g})$ were separated by $12 \%$ SDS-PAGE and transferred onto PVDF membranes (Immobilon-FL membrane, Millipore Company, Massachusetts, USA). Then, the membranes were incubated with 5\% milk in TBST (Tris-buffered saline and $0.5 \%$ Tween 20 ) and incubated overnight with antibodies against Cyp7a1, Cp, or $\beta$-actin $(1: 1000$ Cell Signaling Technology, American) at $4^{\circ} \mathrm{C}$, followed by incubation with goat anti-rabbit IgG HRP (Sangon Biological Technology, Shanghai, China) for $2 \mathrm{~h}$. Finally, the membranes were visualized with a gel documentation system (Aplegen Omega Lum G, American).

2.8. Statistical Analysis. Data are expressed as the mean \pm standard deviation (SD). SPSS software version 16.0 (Chicago, USA) was used for statistical analyses. Results of fasting blood glucose level, body weight, and biochemistry parameters were subjected to one-way analysis of variance (ANOVA). Western blotting results were quantitated by ImageJ software and analyzed by ANOVA. $P<0.05$ was considered statistically significant.

\section{Results}

3.1. Effect of Pioglitazone on the Fasting Blood Glucose Level of Rats. To examine the influence of pioglitazone in vivo, we administered pioglitazone to STZ-induced T2DM rats for a period of 11 weeks. As shown in Figure 1, the fasting blood glucose levels of DM rats and pioglitazone-treated rats were significantly higher compared to those of rats in the control groups at week $0(P<0.01)$. Moreover, after 11 weeks of treatment with pioglitazone, fasting blood glucose levels of pioglitazone-treated rats were significantly lower compared to those of rats in the DM model group $(P<0.05)$.

3.2. Effect of Pioglitazone on Body Weight of Rats. Body weight of rats in the control group, the DM model group, and the pioglitazone-treated group was also recorded in 0 , 1 st, 3rd, 5th, 7th, 9th, and 11th weeks. In the control group, the body weight of rats showed an upward trend from $435.83 \pm 12.97 \mathrm{~g}$ in 0 week to $532.75 \pm 23.18 \mathrm{~g}$ in the 11 th week. However, rats in the DM model group demonstrated a significant loss in the body weight during the 11 weeks. Treatment with pioglitazone slightly increased the body weight of pioglitazone-treated rats and was significantly different compared with the body weights of rats in the DM model group (Figure 2, $P<0.05$ ).

3.3. Effect of Pioglitazone on Biochemistry Parameters of Rats. Rats' plasma lipids were used for the evaluation of biochemistry parameters, including blood urea nitrogen (BUN), total cholesterol (TC), triglyceride (TG), high-density lipoprotein cholesterol (HDL-c), total protein (TP), and albumin (ALB). As shown in Figure 3, levels of the TC, TG, and HDL-c were significantly elevated in response to a high-

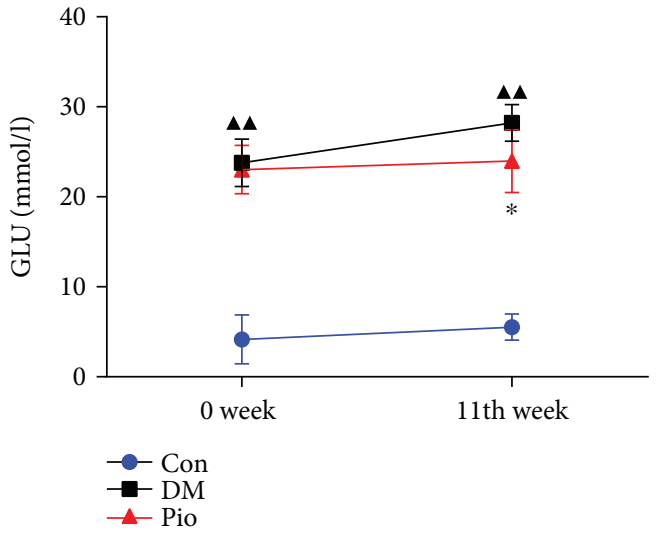

FIGURe 1: Pioglitazone reduces the fasting blood glucose level of DM rats. The fasting blood glucose levels in rats were significantly reduced after treatment with pioglitazone. ${ }^{\boldsymbol{\Delta} \mathbf{\Delta}} P<0.01$, the DM model group compared with the control group; ${ }^{*} P<0.05$, the DM model group compared with the pioglitazone-treated group.

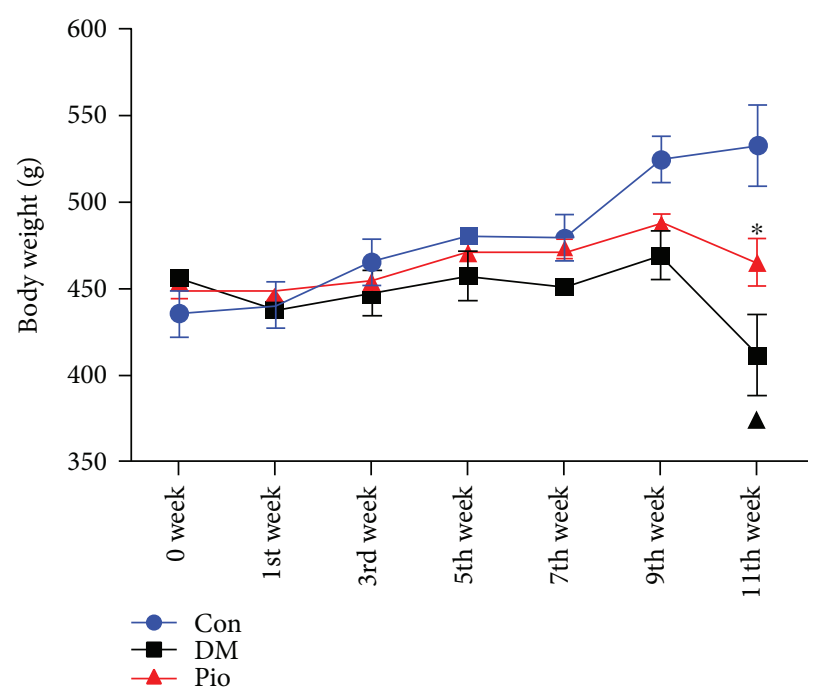

FIgure 2: Pioglitazone alters the body weight of rats. The body weight of rats was recorded in $0,1 \mathrm{st}, 3 \mathrm{rd}, 5 \mathrm{th}, 7 \mathrm{th}$, 9th, and 11th weeks. ${ }^{\wedge} P<0.05$, the DM model group compared with the control group; ${ }^{*} P<0.05$, the DM model group compared with the pioglitazone-treated group.

glucose/high-fat diet $(P<0.01)$. After 11 weeks of pioglitazone treatment, TC, TG, TP, and HDL-c levels significantly decreased compared with rats in the DM model group $(P<0.05 ; P<0.01)$. In previous studies, BUN and ALB measurements were commonly used to determine the liver function and renal function, respectively [18]. We found that levels of BUN and ALB were significantly decreased after pioglitazone treatment $(P<0.01)$, suggesting that pioglitazone has a protective effect on the kidney and liver.

3.4. Differentially Expressed Genes Screened by Expression Profile Chip. By using a 1.5-fold change as the screening criteria for differentially expressed genes, a total of 322 genes were screened in the DM model group and the pioglitazone- 

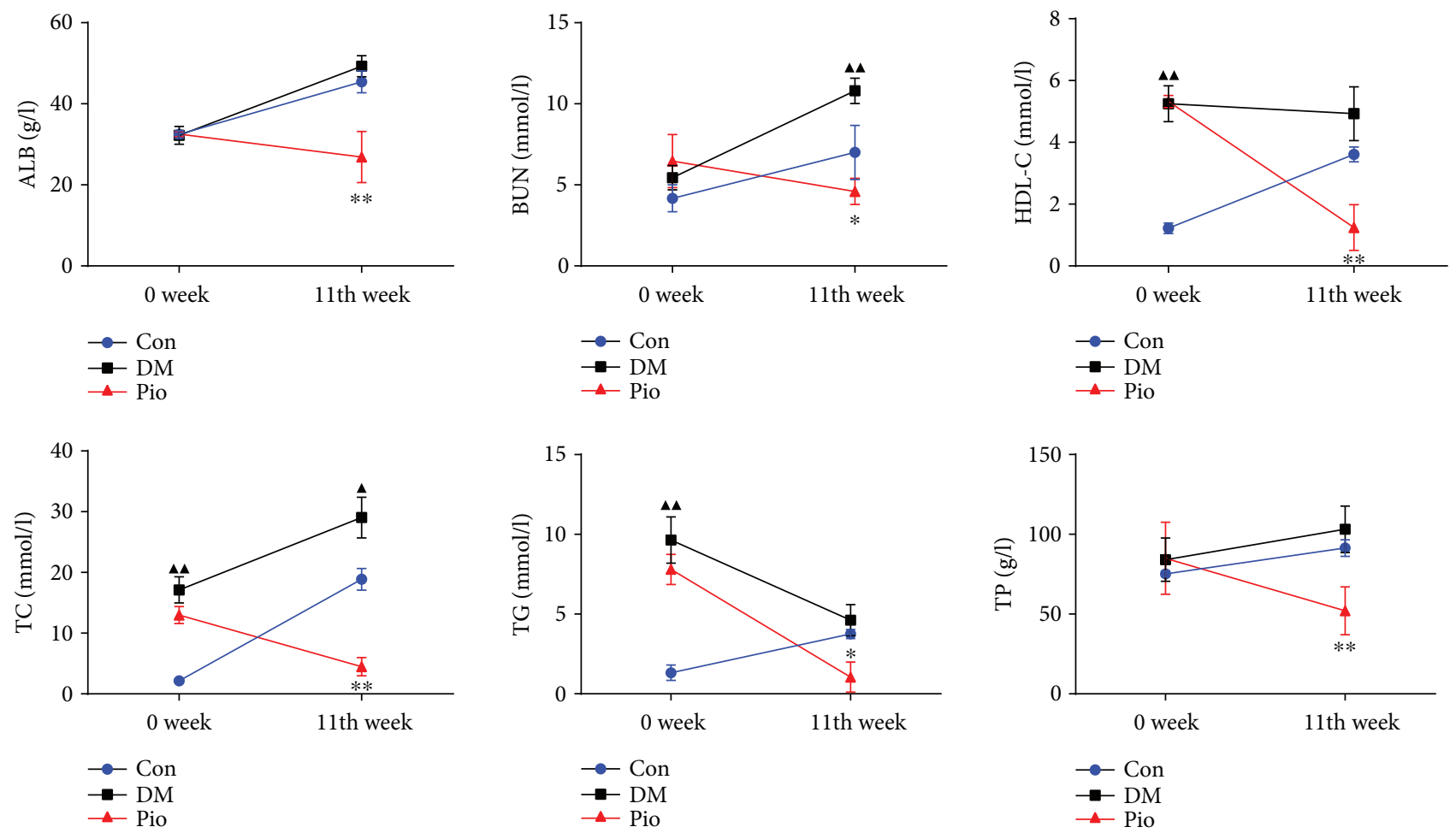

FIGURE 3: Pioglitazone changes blood biochemistry parameters. After treatment with pioglitazone for 11 weeks, the levels of TC, TG, TP, ALB, BUN, and HDL-c were significantly decreased. ${ }^{\boldsymbol{\Delta}} P<0.05 ;{ }^{\wedge \mathbf{\Delta}} P<0.01$, the DM model group compared with the control group; and ${ }^{*} P<0.05$ and ${ }^{* *} P<0.01$, the DM model group compared with the pioglitazone-treated group.

treated group, in which 191 genes were significantly upregulated and 141 genes were significantly downregulated. GO enrichment and KEGG analysis were applied to assay the differentially expressed genes. As shown in Figure 4, pioglitazone treatment had a big impact on the biology process, the molecular function, and the cellular component in the liver. The biology process was affected by pioglitazone treatment, including the response to cAMP, epidermal growth factor receptor signaling pathway, and histone $\mathrm{H} 2 \mathrm{~A}$ acetylation. The pioglitazone-affected molecular function is involved for example in arginine-tRNA ligase activity, pheromone receptor activity, and unfolded protein binding. The cellular component was associated with keratin filament, melanosome, and cytoskeleton. As shown in Table 1, top 10 signaling pathways enriched by KEGG included ribosome biogenesis in eukaryotes, fatty acid metabolism, and protein processing in endoplasmic.

3.5. Differentially Expressed Proteins Screened by iTRAQ. To explore the differentially expressed proteins in the liver, we applied iTRAQ to select potential targets with 1.2-fold change differences as the screening criteria. A total of 204 proteins were found as differentially expressed proteins in the DM model group and the pioglitazone-treated group, of which 96 proteins were significantly upregulated and 108 proteins were significantly downregulated. KEGG pathway enrichment results are presented in Table 2. Proteins with altered expression were categorized according to KEGG pathway analysis and included the retinol metabolism, steroid hormone biosynthesis, and metabolic pathways. Figure 5 presents the gene ontology results. Cellular components affected by pioglitazone mainly included cellular parts, envelope, and extracellular regions. Using GO analysis, major molecular functions intervened by pioglitazone included antioxidant, auxiliary transport protein, and binding. Biological processes identified by GO assay of proteins are presented in Figure 5. The anatomical structure formation, biological adhesion, and biological regulation were among the main affected biological process.

3.6. Transcriptomic and Proteomic Conjoint Analysis of Potential Therapeutic Targets. Further analysis showed 3 molecules were found with the same trend in the pioglitazone-treated group after transcriptomic and proteomic conjoint analysis, namely, Cyp7a1, Cp, and RT1-EC2. Among these targets, the expression levels of $C y p 7 a 1$ and $C p$ were significantly increased, whereas that of $R T 1-E C 2$ decreased (Table 3). Therefore, Cyp7a1, Cp, and RT1-EC2 were identified as potential therapeutic targets in the liver with pioglitazone treatment.

3.7. Validation of Potential Therapeutic Targets. Cyp7a1 (cytochrome P450, family 7, subfamily a, polypeptide I) and $C P$ (Ceruloplasmin) were further validated by qRT-PCR and Western blotting assay. As shown in Figure 6, compared with the DM model group, the gene expressions and protein levels of Cyp7a1 in the pioglitazone-treated group were significantly decreased, while the gene expression level of 


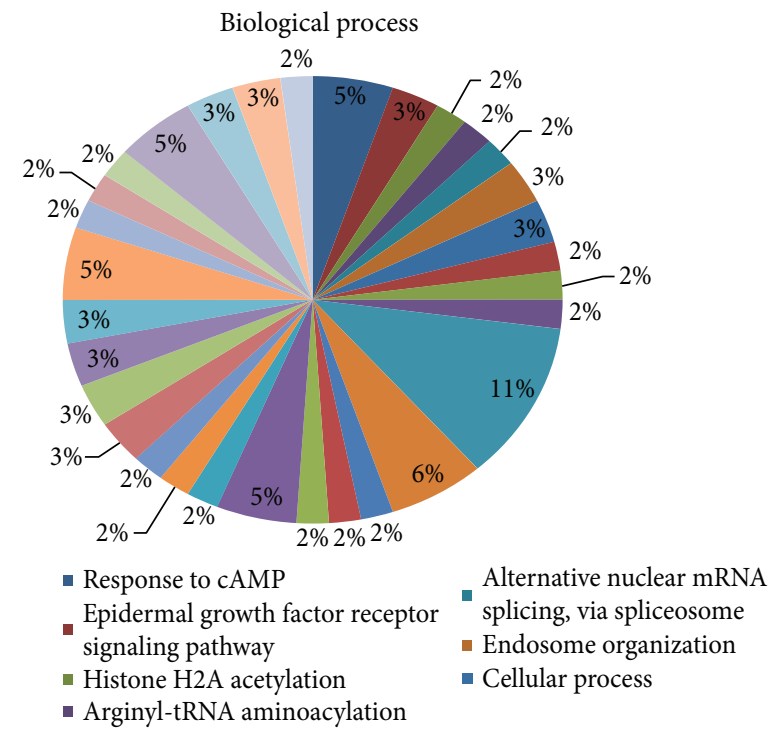

(a)

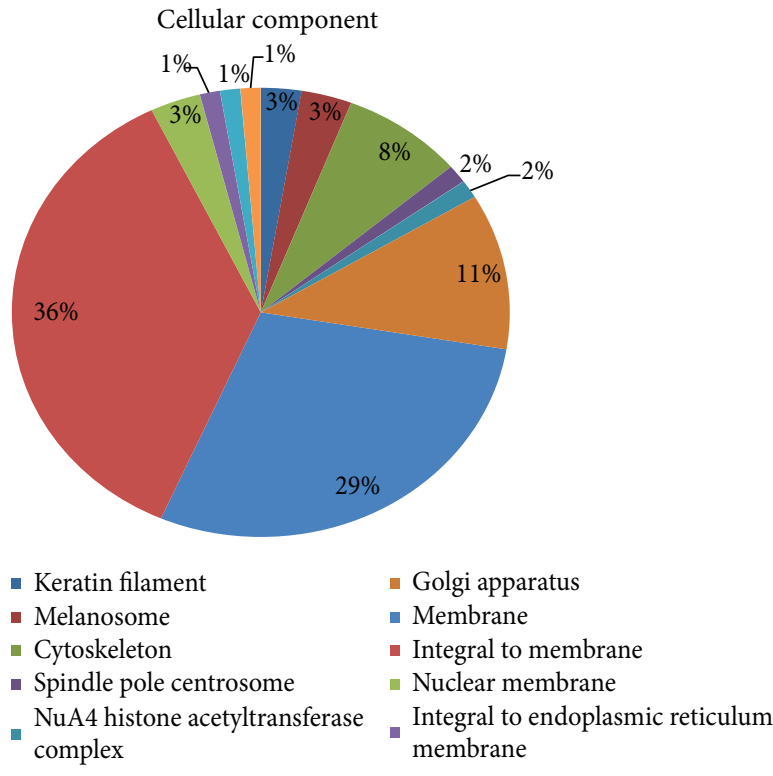

(b)

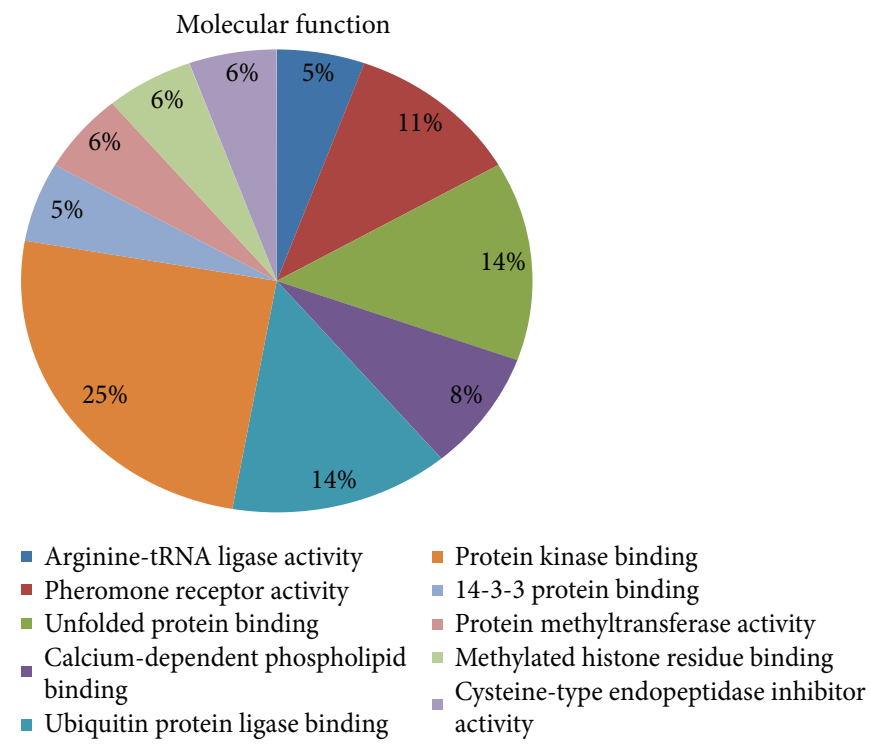

(c)

FIGURE 4: GO analysis of differentially expressed genes after treatment with pioglitazone. Microarray assay results of the DM model group and the pioglitazone-treated group. (a) Biological process. (b) Cellular component. (c) Molecular function.

TABLE 1: KEGG pathway enrichment via gene expression profile chip (DM versus Pio).

\begin{tabular}{lccc}
\hline Number & Pathway ID & Pathway & $P$ value \\
\hline 1 & ko03008 & Ribosome biogenesis in eukaryotes & $2.92 E-02$ \\
2 & ko04977 & Vitamin digestion and absorption & $4.21 E-02$ \\
3 & ko04141 & Protein processing in endoplasmic reticulum & $1.21 E-01$ \\
4 & ko00051 & Fructose and mannose metabolism & $1.48 E-01$ \\
5 & ko00790 & Folate biosynthesis & $1.62 E-01$ \\
6 & ko00071 & Fatty acid metabolism & $1.69 E-01$ \\
7 & ko00970 & Aminoacyl-tRNA biosynthesis & $1.69 E-01$ \\
8 & ko04960 & Aldosterone-regulated sodium reabsorption & $1.69 E-01$ \\
9 & ko03040 & Spliceosome & $1.71 E-01$ \\
10 & ko04612 & Antigen processing and presentation & $1.85 E-01$ \\
\hline
\end{tabular}


TABLE 2: KEGG pathway enrichment via iTRAQ (DM versus Pio).

\begin{tabular}{lccr}
\hline Number & Pathway ID & Pathway & $P$ value \\
\hline 1 & ko00830 & Retinol metabolism & $4.44 E-09$ \\
2 & ko00140 & Steroid hormone biosynthesis & $2.49 E-06$ \\
3 & ko01100 & Metabolic pathways & $8.68 E-06$ \\
4 & ko00980 & Metabolism of xenobiotics by cytochrome P450 & $1.54 E-05$ \\
5 & ko00982 & Drug metabolism-cytochrome P450 & $1.62 E-05$ \\
6 & ko04976 & Bile secretion & $7.75 E-05$ \\
7 & ko03320 & PPAR signaling pathway & $4.96 E-04$ \\
8 & ko00590 & Arachidonic acid metabolism & $6.60 E-04$ \\
9 & ko00920 & Sulfur metabolism & $2.41 E-03$ \\
10 & ko00983 & Drug metabolism-other enzymes & $4.72 E-03$ \\
\hline
\end{tabular}

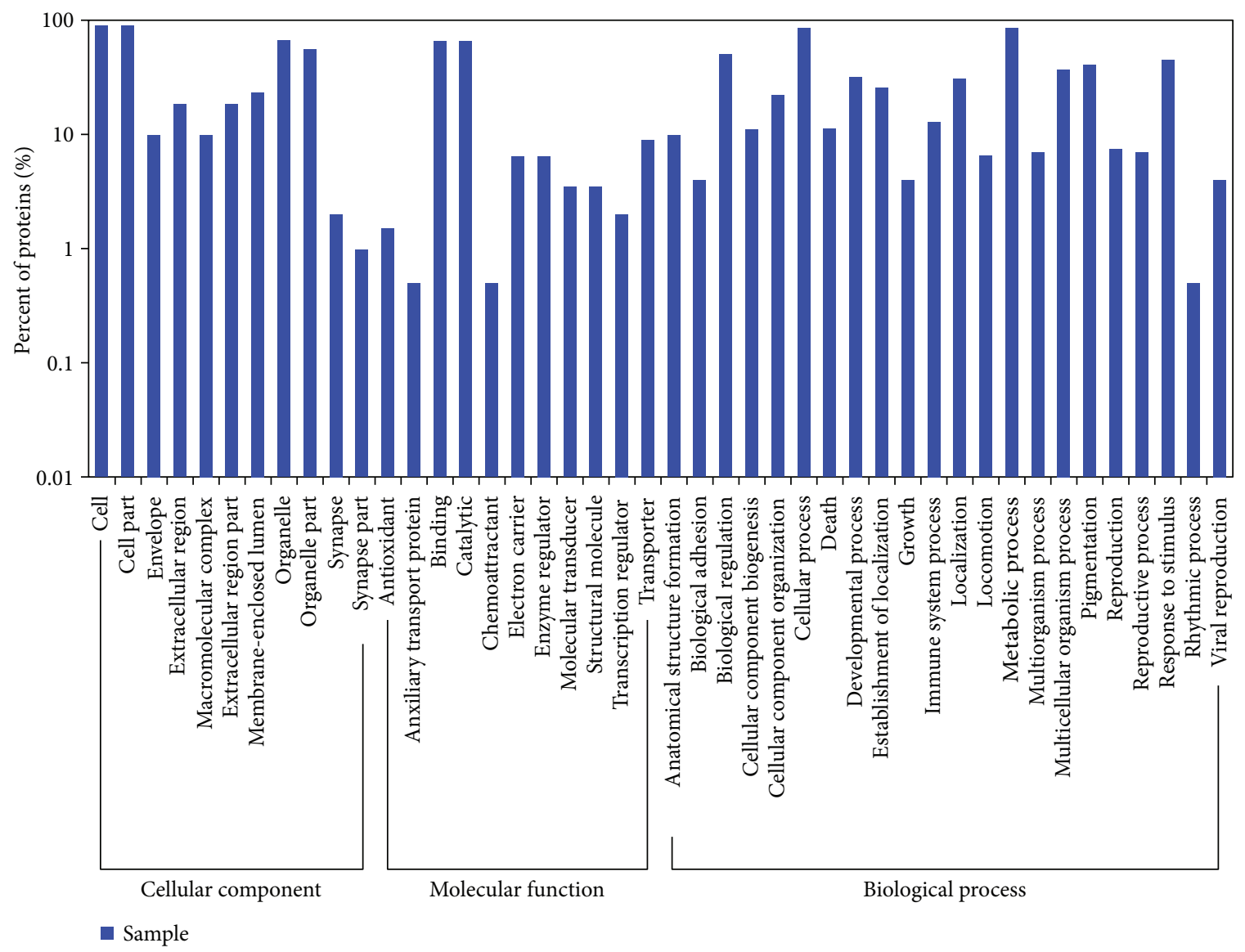

FIGURE 5: GO analysis of differentially expressed proteins after treatment with pioglitazone. iTRAQ assay results of the DM model group and the pioglitazone-treated group.

TABLE 3: Transcriptomic and proteomic conjoint analysis of potential targets.

\begin{tabular}{lccccc}
\hline \multirow{2}{*}{ Gene symbol } & \multicolumn{2}{c}{ Transcriptomics } & \multicolumn{2}{c}{ Proteomics } \\
& Log $($ Pio versus DM $)$ & $\log ($ Con versus DM $)$ & Pio versus DM & Con versus DM & Regulation \\
\hline Cyp7a1 & -0.06 & -1.52 & 0.488 & 0.361 & Down \\
Cp & -0.17 & -1.15 & 0.826 & 0.648 & Down \\
RT1-EC2 & 0.21 & 0.24 & 1.255 & 1.204 & Up \\
\hline
\end{tabular}



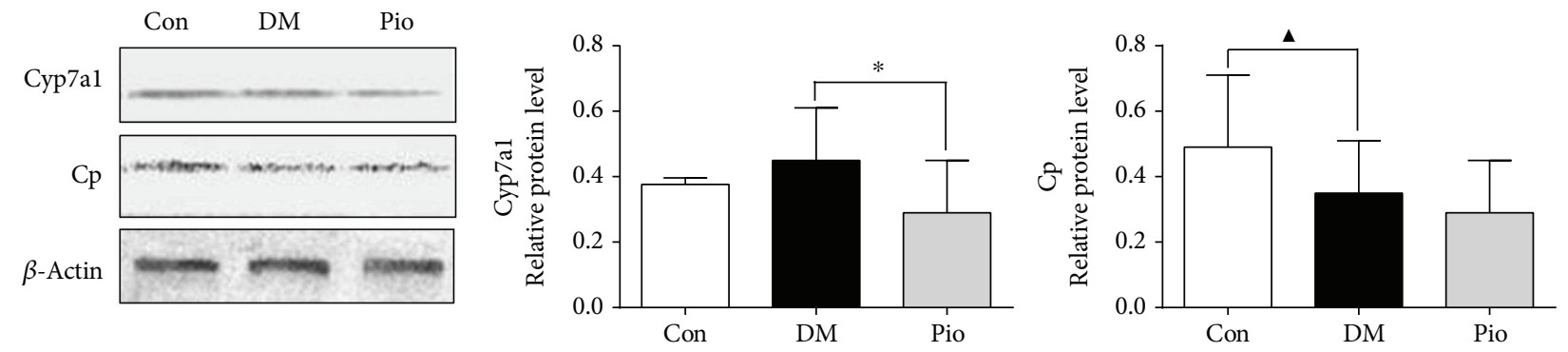

(a)
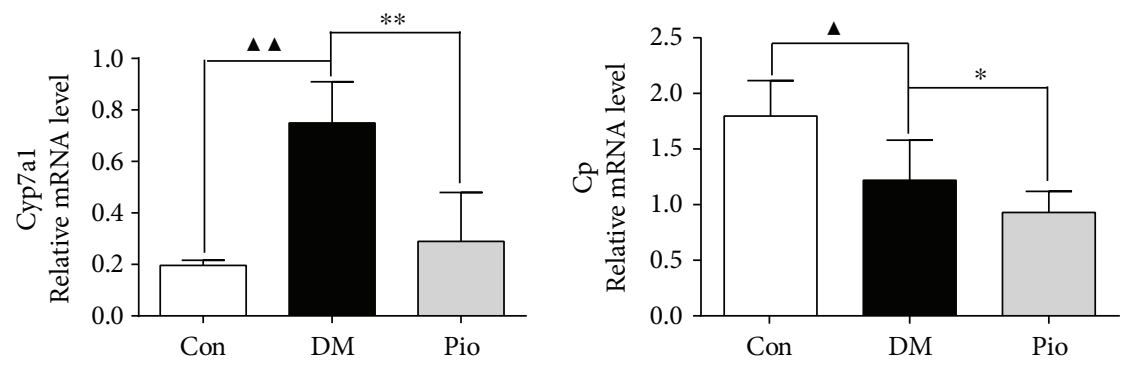

(b)

Figure 6: Data verified by RT-qPCR and Western blotting. (a) Gene expression detected by RT-qPCR. (b) Protein expression detected by Western blotting. ${ }^{\boldsymbol{\Delta}} P<0.05$; ${ }^{\boldsymbol{\Lambda}} P<0.01$, the DM model group compared with the control group; and ${ }^{*} P<0.05$ and ${ }^{* *} P<0.01$, the DM model group compared with the pioglitazone-treated group.

$C p$ in the pioglitazone-treated group was also significantly downregulated after treatment with pioglitazone for 11 weeks. The results confirmed the former microarray and iTRAQ findings.

\section{Discussion}

T2DM is characterized by chronic hyperglycemia due to worsening insulin resistance, which is a hallmark of type 2 diabetes [19]. Practically, insulin resistance exists throughout the course of diabetes; thus, enhancing insulin sensitivity is a key strategy for treatment [20]. Pioglitazone is an antidiabetic drug for the clinical treatment of T2DM and has been used over the past 50 years. It is known to improve insulin sensitivity, glycaemic control, hypertension, dyslipidemia, and microalbuminuria acting on PPAR- $\gamma[21,22]$.

In the present study, we established T2DM model rats with STZ and a high-glucose/high-fat diet according to the Reed method [23]. Pioglitazone was given by gavage to treat T2DM rats. After treatment with pioglitazone, fasting blood glucose levels were significantly lower compared to that in the model group. In addition, the levels of TC, TG, TP, ALB, BUN, and HDL-c were also significantly reduced.

Microarray and iTRAQ, high-throughput bioinformatics technologies, are widely used in drug screening, new drug development, and disease diagnosis [24-26]. The liver is regarded as a metabolic center and plays vital important roles in material metabolism and energy metabolism by regulating the metabolism of numerous molecules, such as carbohydrates, lipids, proteins, and hormones [27]. Hence, in the present study, we selected the liver as the target organ to study the changes of genes and proteins after pioglitazone treatment. Based on the results from microarray and iTRAQ analysis, $C y p 7 a 1, C p$, and RT1-EC2 were selected as the most significantly differentially expressed targets.

Ceruloplasmin $(C p)$ is a ferroxidase enzyme synthesized in the liver and is involved in carrying copper in the blood and in addition plays a role in iron metabolism [28, 29]. $C p$ is also used as an index to assess hepatic disease; the expression level of $C p$ dropped due to reduced synthesizing capabilities after liver damage [30]. RT1-EC2 is a class $1 \mathrm{~b}$ gene of the rat major histocompatibility complex, which is reported to be responsible for the development of diabetes in rats $[31,32]$. Cyp7a1 belongs to the cytochrome $\mathrm{P} 450$ family of enzymes and encodes cholesterol 7a-hydroxylase, which is the rate-limiting enzyme in bile acid biosynthesis from cholesterol [33]. Previous studies have reported that Cyp7a1 is associated with circulating cholesterol concentrations in response to various dietary interventions [34]. Furthermore, numerous studies have reported that Cyp7a1 plays a vitally important role in modulation of bile acid, lipid, and glucose homeostasis [35-37]. Therefore, Cyp7a1 may serve as a potential candidate therapeutic node for diabetes.

\section{Conclusions}

In summary, our study demonstrated that pioglitazone is a multitarget antidiabetic drug affecting numerous genes involved in lipid and cholesterol metabolism pathways. Through microarray and iTRAQ analyses, $C y p 7 a 1, C p$, and RT1-EC2 were the most differentially expressed molecules in the DM group and the pioglitazone-treated group. Moreover, our study provides valuable data that may help in elucidating the underlying mechanism of pioglitazone in T2DM. 


\section{Abbreviations}

$\begin{array}{ll}\text { TC: } & \text { Total cholesterol } \\ \text { TG: } & \text { Triglyceride } \\ \text { TP: } & \text { Total protein } \\ \text { ALB: } & \text { Albumin } \\ \text { BUN: } & \text { Blood urea nitrogen } \\ \text { HDL-c: } & \text { High-density lipoprotein cholesterol } \\ \text { DM: } & \text { Diabetes mellitus } \\ \text { T2DM: } & \text { Type 2 diabetes mellitus } \\ \text { GO: } & \text { Gene ontology } \\ \text { KEGG: } & \text { Kyoto Encyclopedia of Genes and Genomes } \\ \text { Cyp7a1: } & \text { Cytochrome P450, family 7, subfamily a, } \\ & \text { polypeptide I } \\ \text { Cp: } & \text { Ceruloplasmin } \\ \text { RT1-EC2: } & \text { RT1 class Ib, locus EC2. }\end{array}$

\section{Conflicts of Interest}

The authors declare that they have no conflicts of interest.

\section{Acknowledgments}

The present study was supported by grants from National Great New Drug Research and Development (no. 2012ZX09503001-001) and Traditional Chinese Medicine Open Funds of Zhejiang Chinese Medical University (no. 752223A00201/005/019).

\section{References}

[1] L. Luo, W. H. Zhou, J. J. Cai et al., "Gene expression profiling identifies downregulation of the neurotrophin-MAPK signaling pathway in female diabetic peripheral neuropathy patients," Journal of Diabetes Research, vol. 2017, Article ID 8103904, 11 pages, 2017.

[2] J. S. Skyler, G. L. Bakris, E. Bonifacio et al., "Differentiation of diabetes by pathophysiology, natural history, and prognosis," Diabetes, vol. 66, no. 2, pp. 241-255, 2017.

[3] Y. Luo, S. K. Paul, X. Zhou et al., "Design, and baseline characteristics of Beijing prediabetes reversion program: a randomized controlled clinical trial to evaluate the efficacy of lifestyle intervention and/or pioglitazone in reversion to normal glucose tolerance in prediabetes," Journal of Diabetes Research, vol. 2017, Article ID 7602408, 2017.

[4] J. Zhang, X. J. Wang, X. Liu et al., "Design, sythesis and evaluation of a series of 3- or 4-alkoxy substituted phenoxy derivatives as PPARs agonists," Oncotarget, vol. 8, no. 13, pp. 20766-20783, 2017.

[5] Y. Zhang, J. Yu, V. Grachtchouk et al., "Genomic binding of PAX8-PPARG fusion protein regulates cancer-related pathways and alters the immune landscape of thyroid cancer," Oncotarget, vol. 8, no. 4, pp. 5761-5773, 2017.

[6] C. E. Shannon, G. Daniele, C. Galindo, M. A. Abdul-Ghani, R. A. DeFronzo, and L. Norton, "Pioglitazone inhibits mitochondrial pyruvate metabolism and glucose production in hepatocytes," FEBS Journal, vol. 284, no. 3, pp. 451-465, 2017.

[7] S. Yandrapalli and W. S. Aronow, "Cardiovascular benefits of the newer medications for treating type 2 diabetes mellitus," Journal of Thoracic Disease, vol. 9, no. 7, pp. 21242134, 2017.

[8] L. Zhao, Y. Pan, K. Peng et al., "Inhibition of $11 \beta$-HSD1 by LG13 improves glucose metabolism in type 2 diabetic mice," Journal of Molecular Endocrinology, vol. 55, no. 2, pp. 119131, 2015.

[9] A. L. Hopkins, "Network pharmacology: the next paradigm in drug discovery," Nature Chemical Biology, vol. 4, no. 11, pp. 682-690, 2008.

[10] A. L. Hopkins, "Network pharmacology," Nature Biotechnology, vol. 25, no. 10, pp. 1110-1111, 2007.

[11] J. Fang, L. Wang, T. Wu et al., "Network pharmacologybased study on the mechanism of action for herbal medicines in Alzheimer treatment," Journal of Ethnopharmacology, vol. 196, pp. 281-292, 2017.

[12] C. Perrino, A. L. Barabasi, G. Condorelli et al., "Epigenomic and transcriptomic approaches in the post-genomic era: path to novel targets for diagnosis and therapy of the ischaemic heart? Position paper of the European Society of Cardiology Working Group on cellular biology of the heart," Cardiovascular Research, vol. 113, no. 7, pp. 725-736, 2017.

[13] F. Sun, S. Chai, L. Li et al., "Effects of glucagon-like peptide-1 receptor agonists on weight loss in patients with type 2 diabetes: a systematic review and network meta-analysis," Journal of Diabetes Research, vol. 2015, Article ID 157201, 9 pages, 2015.

[14] B. Li, X. Jin, H. Meng et al., "Morin promotes prostate cancer cells chemosensitivity to paclitaxel through miR-155/GATA3 axis," Oncotarget, vol. 8, no. 29, pp. 47849-47860, 2017.

[15] H. Cao, H. Yu, Y. Feng, L. Chen, and F. Liang, "Curcumin inhibits prostate cancer by targeting PGK1 in the FOXD3/ miR-143 axis," Cancer Chemotherapy and Pharmacology, vol. 79, no. 5, pp. 985-994, 2017.

[16] A. Madsen, O. Bozickovic, J. I. Bjune, G. Mellgren, and J. V. Sagen, "Metformin inhibits hepatocellular glucose, lipid and cholesterol biosynthetic pathways by transcriptionally suppressing steroid receptor coactivator 2 (SRC-2)," Scientific Reports, vol. 5, no. 1, article 16430, 2015.

[17] Y. S. Wu, Y. T. Chen, Y. T. Bao et al., "Identification and verification of potential therapeutic target genes in Berberinetreated Zucker diabetic fatty rats through bioinformatics analysis," PLoS One, vol. 11, no. 11, article e0166378, 2016.

[18] J. A. Messana, S. S. Schwartz, and R. R. Townsend, "An evidence-based practice-oriented review focusing on canagliflozin in the management of type 2 diabetes," Vascular Health and Risk Management, vol. 13, pp. 43-54, 2017.

[19] P. Zizkova, M. Stefek, L. Rackova, M. Prnova, and L. Horakova, "Novel quercetin derivatives: from redox properties to promising treatment of oxidative stress related diseases," Chemico-Biological Interactions, vol. 265, pp. 36-46, 2017.

[20] X. T. Feng, S. Y. Tang, Y. X. Jiang, and W. Zhao, “Anti-diabetic effects of Zhuoduqing formula, a Chinese herbal decoction, on a rat model of type 2 diabetes," African Journal of Traditional Complementary and Alternative Medicines, vol. 14, no. 3, pp. 42-50, 2017.

[21] X. Z. Zhang, H. Imachi, J. Y. Lyu et al., "Prolactin regulatory element-binding protein is involved in suppression of the adiponectin gene in vivo," Journal of Endocrinological Investigation, vol. 40, no. 4, pp. 437-445, 2017. 
[22] H. W. Liao, J. L. Saver, Y. L. Wu, T. H. Chen, M. Lee, and B. Ovbiagele, "Pioglitazone and cardiovascular outcomes in patients with insulin resistance, pre-diabetes and type 2 diabetes: a systematic review and meta-analysis," BMJ Open, vol. 7, no. 1, article e013927, 2017.

[23] M. J. Reed, K. Meszaros, L. J. Entes et al., "A new rat model of type 2 diabetes: the fat-fed, streptozotocin-treated rat," Metabolism, vol. 49, no. 11, pp. 1390-1394, 2000.

[24] J. Yu, X. Li, C. Zhong et al., "High-throughput proteomics integrated with gene microarray for discovery of colorectal cancer potential biomarkers," Oncotarget, vol. 7, no. 46, pp. 75279-75292, 2016.

[25] H. Lu, Y. Yang, E. M. Allister, N. Wijesekara, and M. B. Wheeler, "The identification of potential factors associated with the development of type 2 diabetes: a quantitative proteomics approach," Molecular \& Cellular Proteomics, vol. 7, no. 8, pp. 1434-1451, 2008.

[26] C. H. White, H. E. Johnston, B. Moesker et al., "Mixed effects of suberoylanilide hydroxamic acid (SAHA) on the host transcriptome and proteome and their implications for HIV reactivation from latency," Antiviral Research, vol. 123, pp. 78-85, 2015.

[27] H. Yoshida, R. Tsuhako, T. Atsumi et al., "Naringenin interferes with the anti-diabetic actions of pioglitazone via pharmacodynamic interactions," Journal of Natural Medicines, vol. 71, no. 2, pp. 442-448, 2017.

[28] M. G. Skalnaya, A. V. Skalny, and A. A. Tinkov, "Serum copper, zinc, and iron levels, and markers of carbohydrate metabolism in postmenopausal women with prediabetes and type 2 diabetes mellitus," Journal of Trace Elements in Medicine and Biology, vol. 43, pp. 46-51, 2017.

[29] C. Gluhovschi, G. Gluhovschi, L. Petrica et al., "Urinary biomarkers in the assessment of early diabetic nephropathy," Journal of Diabetes Research, vol. 2016, Article ID 4626125, 13 pages, 2016.

[30] R. Squitti, A. J. Mendez, I. Simonelli, and C. Ricordi, “Diabetes and Alzheimer's disease: can elevated free copper predict the risk of the disease?," Journal of Alzheimers Disease, vol. 56, no. 3, pp. 1055-1064, 2017.

[31] E. Rothermel, L. Heine, W. Wurst, and E. Gunther, "Characterization of a class Ib gene of the rat major histocompatibility complex," Immunogenetics, vol. 38, no. 2, pp. 82-91, 1993.

[32] N. Yokoi, C. Hayashi, Y. Fujiwara, H. Y. Wang, and S. Seino, "Genetic reconstitution of autoimmune type 1 diabetes with two major susceptibility genes in the rat," Diabetes, vol. 56, no. 2, pp. 506-512, 2007.

[33] Q. Wu, X. Zhang, M. Zhong et al., "Effects of bariatric surgery on serum bile acid composition and conjugation in a diabetic rat model," Obesity Surgery, vol. 26, no. 10, pp. 2384-2392, 2016.

[34] Y. Wang, S. V. Harding, P. Eck et al., "High-molecular-weight beta-glucan decreases serum cholesterol differentially based on the CYP7A1 rs3808607 polymorphism in mildly hypercholesterolemic adults," Journal of Nutrition, vol. 146, no. 4, pp. 720 727, 2016.

[35] M. Zhou, J. Luo, M. Chen et al., "Mouse species-specific control of hepatocarcinogenesis and metabolism by FGF19/ FGF15," Journal of Hepatology, vol. 66, no. 6, pp. 1182-1192, 2017.
[36] D. Y. Saad, M. M. Soliman, A. A. Baiomy, M. H. Yassin, and H. B. El-Sawy, "Effects of karela (bitter melon; Momordica charantia) on genes of lipids and carbohydrates metabolism in experimental hypercholesterolemia: biochemical, molecular and histopathological study," BMC Complementary and Alternative Medicine, vol. 17, no. 1, p. 319, 2017.

[37] J. S. Bae, J. M. Park, J. Lee et al., "Amelioration of nonalcoholic fatty liver disease with NPC1L1-targeted IgY or n-3 polyunsaturated fatty acids in mice," Metabolism, vol. 66, pp. 32-44, 2017. 


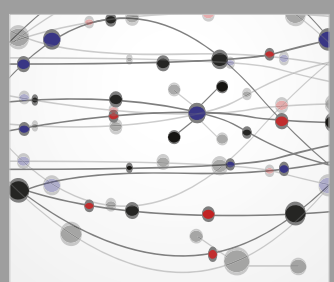

The Scientific World Journal
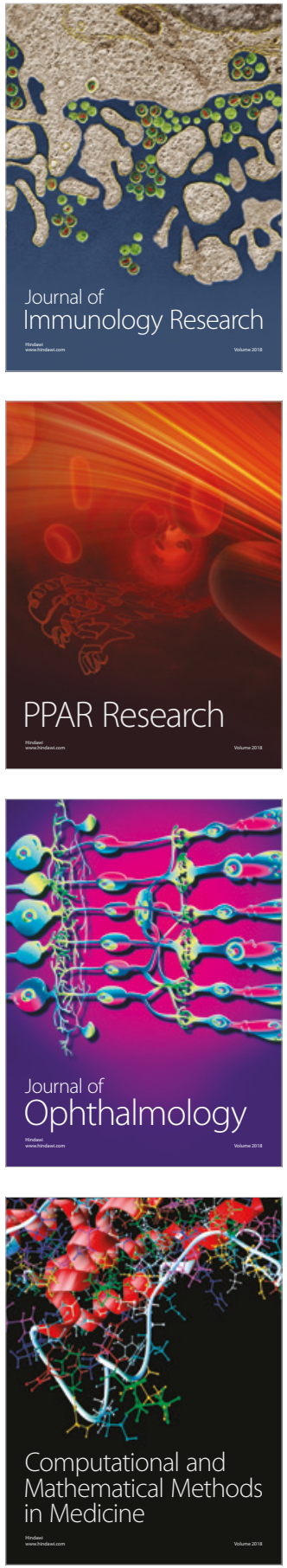

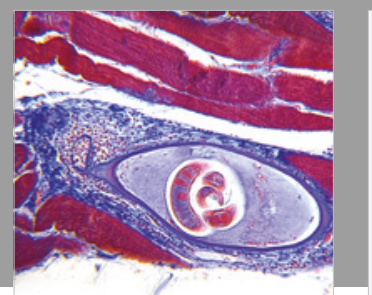

Gastroenterology Research and Practice

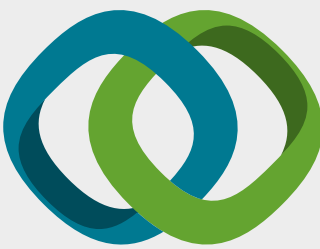

\section{Hindawi}

Submit your manuscripts at

www.hindawi.com
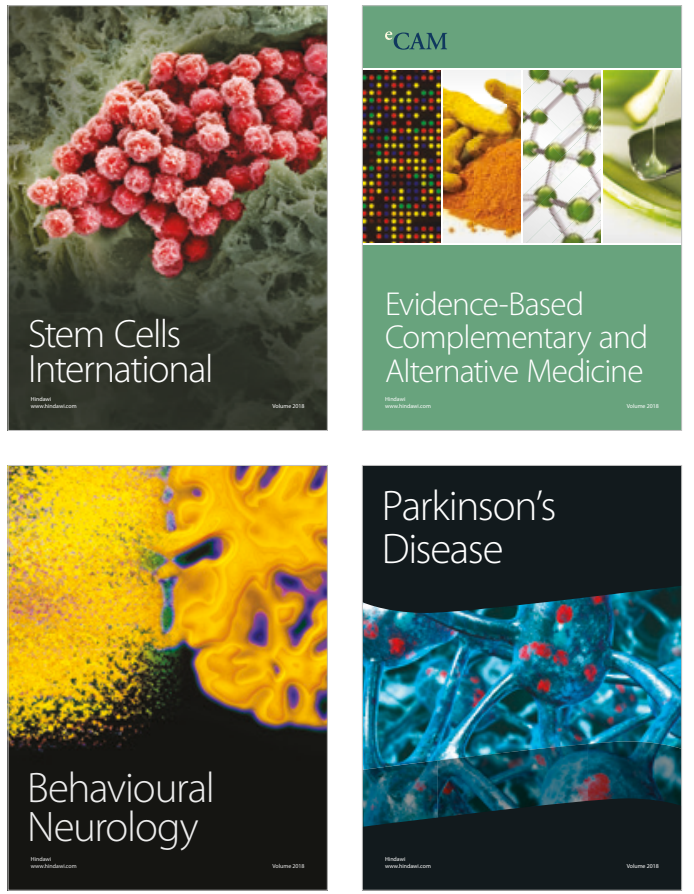

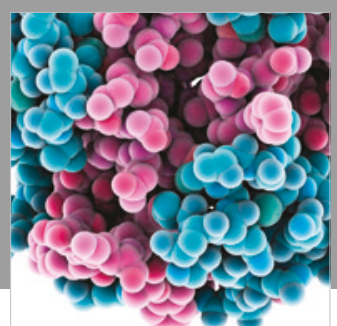

ournal of

Diabetes Research

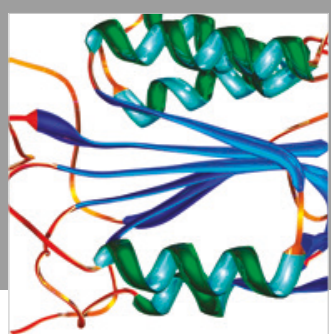

Disease Markers
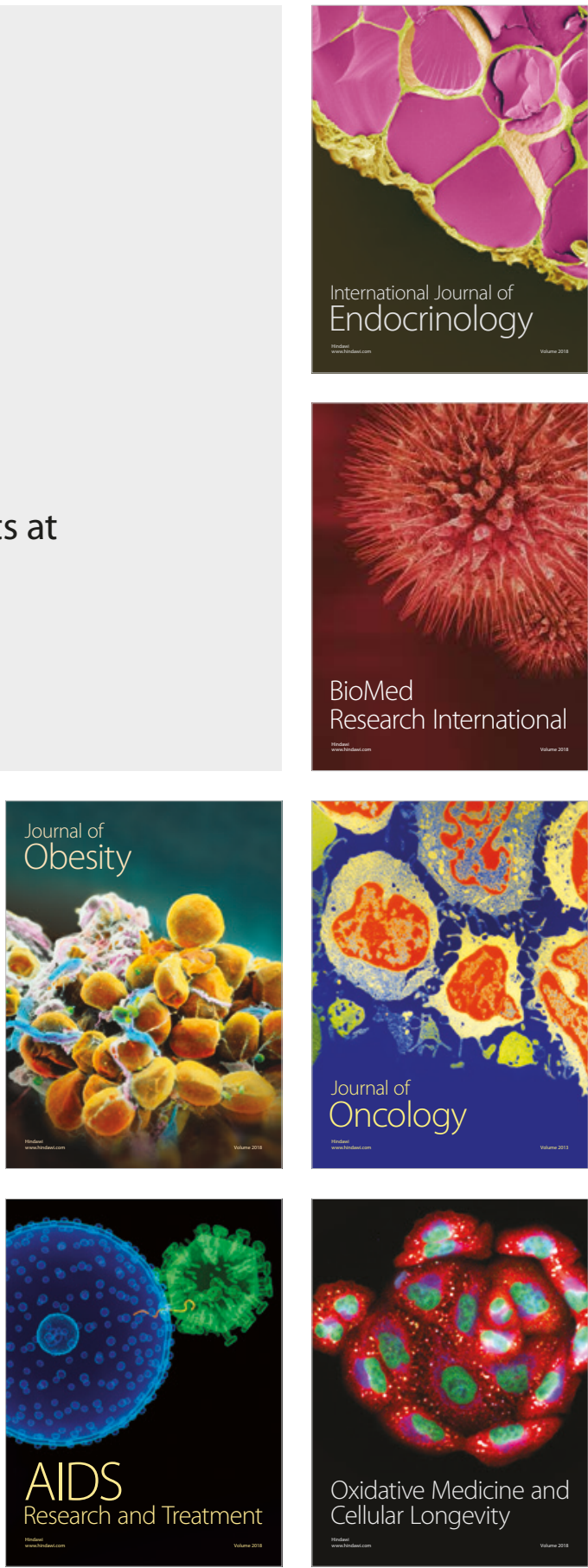